TITLE:

\title{
Monitoring of interarea oscillation mode by synchronized phasor measurement
}

\section{AUTHOR(S):}

Kakimoto, N; Sugumi, M; Makino, T; Tomiyama, K

\section{CITATION:}

Kakimoto, N ... [et al]. Monitoring of interarea oscillation mode by synchronized phasor measurement. IEEE TRANSACTIONS ON POWER SYSTEMS 2006, 21(1): 260-268

\section{ISSUE DATE:}

2006-02

URL:

http://hdl.handle.net/2433/50075

\section{RIGHT:}

(c)2006 IEEE. Personal use of this material is permitted. However, permission to reprint/republish this material for advertising or promotional purposes or for creating new collective works for resale or redistribution to servers or lists, or to reuse any copyrighted component of this work in other works must be obtained from the IEEE. 


\title{
Monitoring of Interarea Oscillation Mode by Synchronized Phasor Measurement
}

\author{
Naoto Kakimoto, Member, IEEE, Masahiro Sugumi, Tohru Makino, and Katsuyuki Tomiyama
}

\begin{abstract}
There is a low-frequency interarea oscillation mode in middle and western $60-\mathrm{Hz}$ areas of Japan. Its stability determines available transfer capacity of a tie-line. We propose a method of monitoring its stability by phasor measurements synchronized using the global positioning system. The phase angle between two places deviates randomly. However, its Fourier spectrum shows a clear peak corresponding to the interarea mode. We estimate its frequency and damping from the spectrum. The estimation results agree well with those obtained by curve fitting for large disturbances. It is also possible to estimate the eigenvector. We examine how the eigenvalue varies with the total generation and power flow in the areas.
\end{abstract}

Index Terms-Global positioning system (GPS), interarea oscillation mode, monitoring, power system stability, synchronized phasor measurement.

\section{INTRODUCTION}

$\mathbf{T}$ HERE is an interarea oscillation mode in middle and western $60-\mathrm{Hz}$ areas of Japan. The mode is stable for small disturbances. However, if its damping is small, it diverges in an oscillatory manner for large disturbances [1]. The mode restricts power transmission in the areas. Available transfer capacity (ATC) is usually calculated by offline numerical simulation. However, it is desired to present the capacity in real time in deregulated electricity markets. As a step to this target, we consider to monitor the eigenvalue and eigenvector of the interarea mode by measurement. We measure several voltages in the areas and compare their phase angles. Sites of measurement are separated from each other by long distances, but it is required to synchronize the measurements.

Synchronized phasor measurement using the global positioning system (GPS) was developed in the early 1990s, and phasor measuring units (PMUs) are now commercially available [2]-[6]. GPS provides a 1 pulse-per-second (PPS) signal and a time tag, which consists of the year, day, hour, minute, and second. The 1-PPS signal is synchronized to within $1 \mu \mathrm{s}$ of Coordinated Universal Time (UTC). With this signal, PMU's sample voltage or current, calculate phasor, and output with the time tag. They have several important applications. An adaptive out-of-step relaying based on synchronized real-time phase angles was developed in 1993 [7]. Portable power system monitors were used to record and to analyze blackouts of

Manuscript received March 23, 2005; revised September 22, 2005. Paper no. TPWRS-00166-2005.

N. Kakimoto, M. Sugumi, and T. Makino are with Kyoto University, Kyoto 616-8510, Japan (e-mail: kakimoto@kuee.kyoto-u.ac.jp).

K. Tomiyama is with the Kansai Electric Power Company, Osaka 530-0005, Japan.

Digital Object Identifier 10.1109/TPWRS.2005.861960
WSCC in 1996 [8], [9]. Electricite de France started to deploy a coordinated defense plan system against transient instabilities in 1997 [10], [11]. Using relative angles for generator dropping controls was studied [12]. Power system stabilizers with a supplementary input from remote phasor measuring units were also proposed [13].

This paper examines a method of estimating the eigenvalue and eigenvector of the interarea mode in Japan. We use PMUs (NCT2000 Type-A) manufactured by Toshiba [6]. They use 96 samples per cycle of the fundamental frequency to obtain a phasor. Several studies have been made on estimating eigenvalues. Prony analysis is effective to study oscillations following large disturbances [14]. A low-level pseudo-random probing signal is also effective to excite oscillatory modes [15]. Timedomain curve fitting is applied to ambient fluctuations of phase angle in [16]. This method is applicable without disturbing systems, but it yields rather pessimistic results. In this paper, we use a Fourier spectrum to estimate the eigenvalue. This method depends on the random nature of load; therefore, it needs some averaging techniques. We also consider its application to small signal stability monitoring.

First, we describe a measuring method and its results in Section II. Next, we derive a model to explain the results in Section III. A Fourier spectrum of the measurement is considered in Section IV. A method of estimating the eigenvalue is proposed in Section V. The eigenvector is also estimated in Section VI. We verify the effectiveness of the proposed method in Section VII. Lastly, some application to stability monitoring is considered in Section VIII. The results are summarized in Section IX.

\section{Measuring Method}

Fig. 1 shows the middle and western $60-\mathrm{Hz}$ areas of Japan. These areas consist of six electric power companies $S_{1}, S_{2}, \cdots, S_{6}$. There are some 260 large generators in the areas. The total generation capacity is about $120 \mathrm{GW}$.

To monitor the interarea mode, we set two phasor measuring units (PMU) at Fukuoka and Otsu. We also set two PMUs temporarily at Hiroshima and Shizuoka. The distance between Fukuoka and Shizuoka is $1000 \mathrm{~km}$, but the PMUs are synchronized with GPS. We measure common wall outlets of $100 \mathrm{~V}$ on distribution systems. There are several transformers between an outlet and its upper $500-\mathrm{kV}$ bus. The transformers bring some phase shift, a multiple of 30 degrees. However, this phase shift is not a problem since we do not use phase angle itself but rather use its time derivative for our purpose. We have checked that an outlet yields results almost equivalent to its upper $500-\mathrm{kV}$ bus during daily steady-state conditions. 


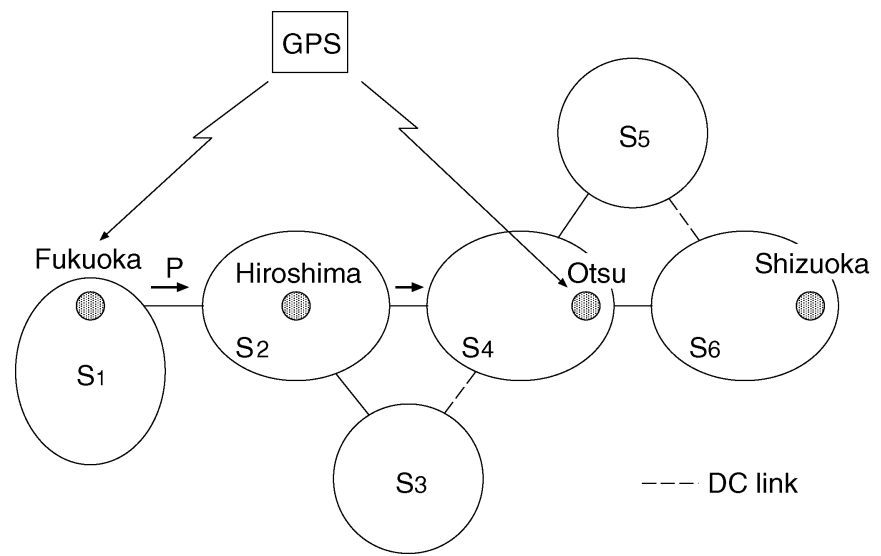

Fig. 1. Middle and western $60-\mathrm{Hz}$ areas of Japan.

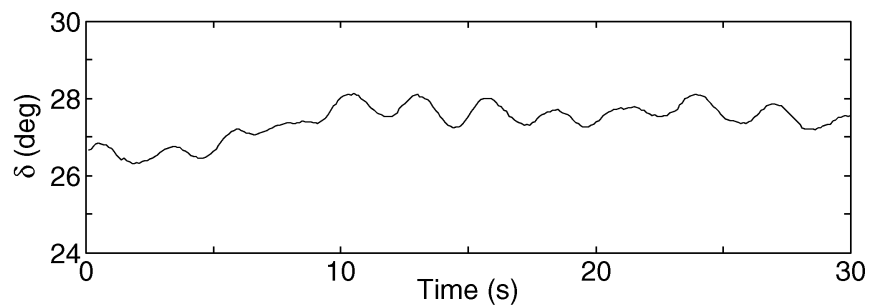

(a)

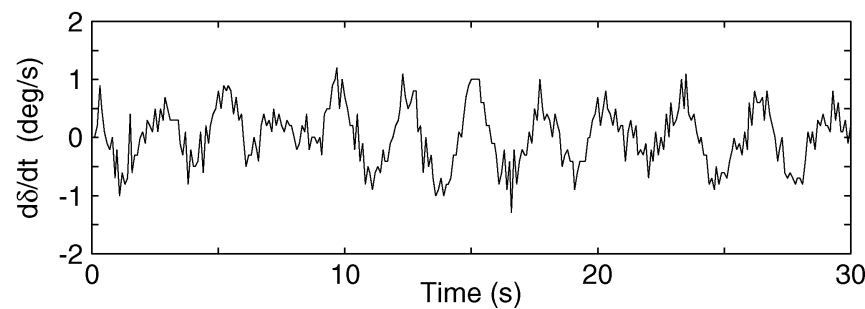

(b)

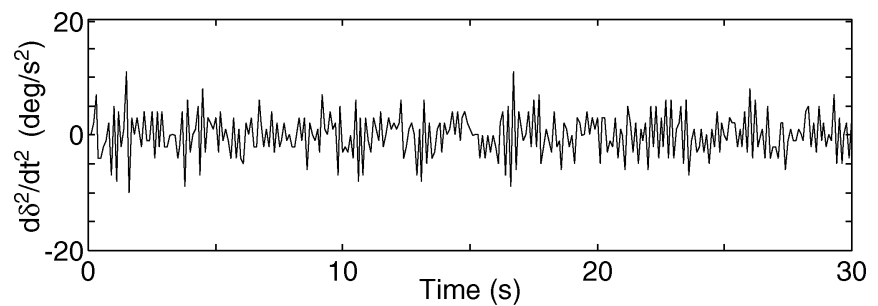

(c)

Fig. 2. Measurement. (a) Phase angle between Fukuoka and Otsu. (b) First time derivative. (c) Second time derivative.

Fig. 2(a) shows time variation of the phase angle $\delta$ between Fukuoka and Otsu measured at 15:00 on July 28, 2004, where the phase angle of Otsu is taken as reference. Sampling time, denoted by $\Delta t$, is $0.1 \mathrm{~s}$, and 36000 samples of the phase angle are obtained every hour. The phase angle is about 27 degrees, but it is always fluctuating. We can observe oscillations of $0.37 \mathrm{~Hz}$. They correspond to the interarea mode that we monitor. However, due to constant disturbance, the oscillations do not monotonically decay. They grow sometime and decay another time. It is difficult to determine their damping constant. Fig. 2(b) and (c) are the first and the second time derivatives of the phase angle. They are not smooth but contain many pulses, especially in (c).

\section{SYSTEM MODEL}

To explain the result in Fig. 2, we assume the phase angle $\delta$ is described by a second order equation as follows:

$$
\frac{d^{2} \delta}{d t^{2}}+2 d \frac{d \delta}{d t}+\omega^{2} \delta=s_{o}+\sum_{i=1}^{n} s_{i}\left(t_{i}\right)
$$

where $d$ and $\omega$ are the damping constant and undamped natural angular frequency of the interarea mode, $s_{o}$ is a constant, $s_{i}$ is a step change that occurs at time $t_{i}$, and $n$ is the number of changes. The term $s_{i}$ represents switching on or off of a load. The total load changes with these switchings.

The time $t_{i}(i=1, \ldots, n)$ is assumed to distribute uniformly in a time interval

$$
0 \leq t<m \Delta t
$$

where $m$ is the number of samples. If $m=2^{15}=32768$, and $\Delta t=0.1 \mathrm{~s}$, the time interval becomes $54.6 \mathrm{~min}$. We generate $t_{i}$ by the congruence method [17]. We set $j_{1}=1$, for example, and calculate $j_{i}$ by

$$
j_{i+1} \equiv p \times j_{i} \quad(\bmod m)
$$

where $p$ is a prime number, and $0 \leq j_{i}<m$. As an example, if $p=8009, j_{i}(i=1,2, \ldots)$ becomes

$$
1,8009,17105,23705,28321,2793,21361,31289,16705, \ldots
$$

We note that $i<k$ does not mean $j_{i}<j_{k}$. We choose $p$ so that

$$
j_{i} \neq j_{k} \quad \text { for } i \neq k
$$

is satisfied. The time $t_{i}$ is given by

$$
t_{i}=j_{i} \Delta t .
$$

Clearly, $0 \leq t_{i}<m \Delta t$, for $i=1, \cdots, n$.

Differentiating (1) gives

$$
\frac{d^{2} y}{d t^{2}}+2 d \frac{d y}{d t}+\omega^{2} y=\sum_{i=1}^{n} u_{i}\left(t_{i}\right) \equiv u(t)
$$

where $y \equiv d \delta / d t$, and $u_{i} \equiv d s_{i} / d t$ is an impulse that occurs at $t_{i}$. For simplicity, we assume

$$
u_{i}\left(t_{i}\right)=-\bar{u} \text { or }+\bar{u} \quad(i=1, \cdots, n)
$$

where $\bar{u}$ is the average magnitude of the impulses. The \pm sign is chosen evenly. We assign + for odd $i$ and - for even $i$. However, this does not mean that the impulses take + and - alternately as time passes. This is understood from the process of generating $t_{i}$.

Fig. 3(a) shows an example of impulses generated in the above manner, where $\bar{u}=0.2, m=2^{15}, n=0.9 m$, and $p=8009$. Substituting the impulses $u_{i}$ into (3) and numerically integrating it yield a time variation of $y$, as shown in Fig. 3(b), where $d=0.1$, and $\omega=2 \pi \times 0.37$. We can see variation similar to Fig. 2(b). 


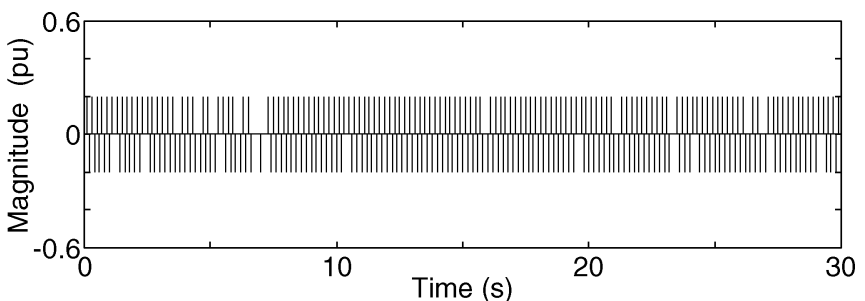

(a)

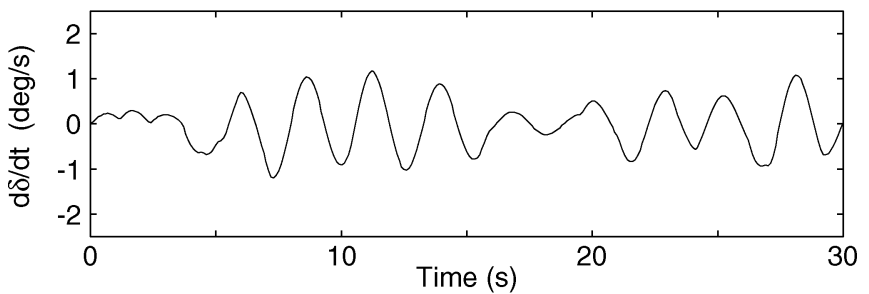

(b)

Fig. 3. Simulation. (a)Impulses $u$. (b) $y \equiv d \delta / d t$.

\section{FOURIER ANALYSIS}

In Fig. 3, we considered time variations of $u$ and $y$. In this section, we consider frequency components contained in the variations. Assume $m$ samples of $u$, for example, are given at $t=j \Delta t,(j=0,1, \ldots, m-1)$, where $m$ is an even number. The values are represented by a finite Fourier series as

$$
u(t)=\sum_{i=0}^{\frac{m}{2}} A_{i} \cos \left(\omega_{i} t+\phi_{i}\right)
$$

where $t=j \Delta t(j=0,1, \ldots, m-1), A_{i}, \omega_{i}, \phi_{i}$ is amplitude, angular frequency, phase, respectively, and

$$
\omega_{i}=\frac{2 \pi}{m \Delta t} i \quad\left(i=0,1, \ldots, \frac{m}{2}\right) .
$$

For details, refer to Appendix A. The amplitude $A_{i}$ and phase $\phi_{i}$ are efficiently calculated with fast Fourier transform (FFT) [18].

Fig. 4 shows Fourier spectra for the simulation results in Fig. 3. The number of samples is $m=2^{15}=32768$, and the sampling time is $\Delta t=0.1 \mathrm{~s}$. We use many samples to obtain accurate results. From (4) and (5), $16385(=m / 2+1)$ frequency components are obtained in

$$
0 \leq \omega_{i} \leq 2 \pi \times 5
$$

The maximum frequency is $5 \mathrm{~Hz}$, which is high enough for our purpose. Fig. 4(a) is the spectrum for the impulses $u$. The amplitudes of the components are not equal but are uniform over all frequencies. Fig. 4(b) shows the spectrum for $d \delta / d t$. Unlike Fig. 4(a), we can see a clear peak around $0.37 \mathrm{~Hz}$. We consider the reason for this.

The sequence of impulses $u$ is represented by (4). Substituting (4) into (3) yields

$$
\frac{d^{2} y}{d t^{2}}+2 d \frac{d y}{d t}+\omega^{2} y=\sum_{i=0}^{\frac{m}{2}} A_{i} \cos \left(\omega_{i} t+\phi_{i}\right)
$$

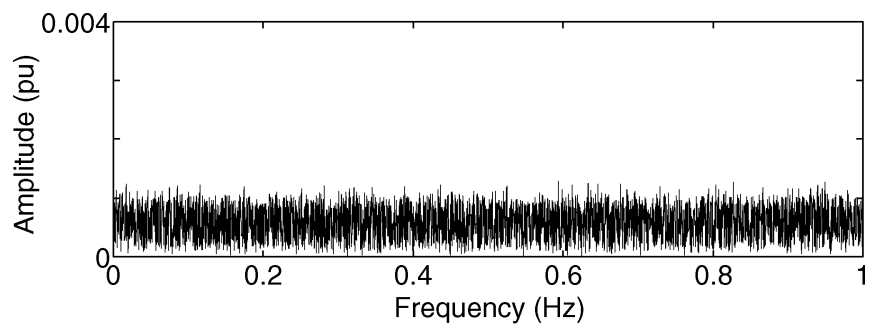

(a)

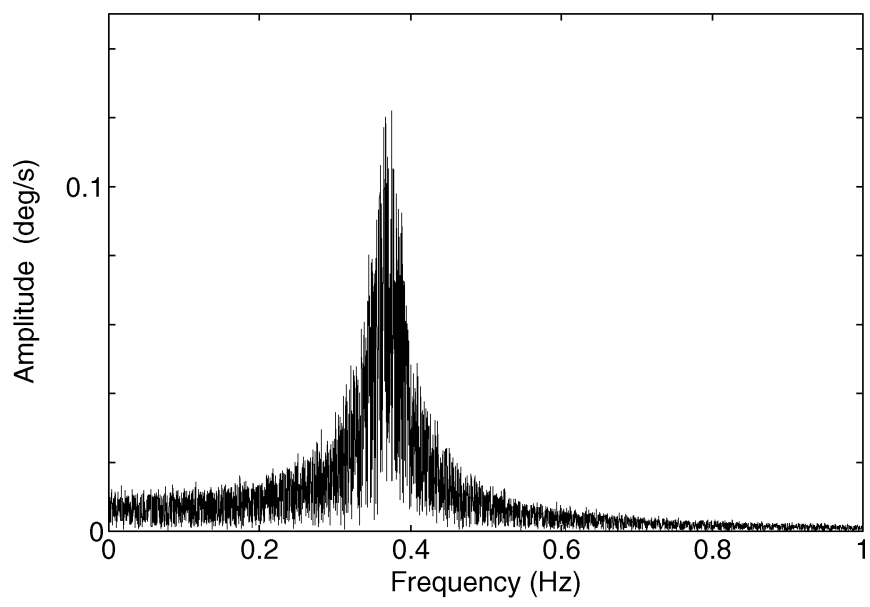

(b)

Fig. 4. Fourier spectrum for simulation. (a) $u$. (b) $y \equiv d \delta / d t$.

The response of the system is

$$
y=\sum_{i=0}^{\frac{m}{2}} c_{i} \cos \left(\omega_{i} t+\phi_{i}+\theta_{i}\right)
$$

where

$$
\begin{aligned}
c_{i} & =\frac{A_{i}}{\sqrt{\left(\omega_{i}^{2}-\omega^{2}\right)^{2}+4 d^{2} \omega_{i}^{2}}} \\
\tan \theta_{i} & =\frac{2 d \omega_{i}}{\left(\omega_{i}^{2}-\omega^{2}\right)} .
\end{aligned}
$$

The amplitudes in Fig. 4(b) correspond to $c_{i}$ in (7). If $A_{i}$ is constant for all $i, c_{i}$ takes its maximum value

$$
c_{i}=\frac{A_{i}}{2 d \omega}{\sqrt{1-\left(\frac{d}{\omega}\right)^{2}}}^{-1} \simeq \frac{A_{i}}{2 d \omega}
$$

at

$$
\omega_{i}=\sqrt{\omega^{2}-2 d^{2}} \simeq \omega
$$

If $d$ is small, the maximum value (9) becomes large.

Fig. 5 shows a Fourier spectrum for the measurement $d \delta / d t$ in Fig. 2(b). The number of samples is $m=2^{15}$, and the sampling time is $\Delta t=0.1 \mathrm{~s}$, again. The amplitude shows variation similar to Fig. 4(b). It takes the maximum value around $0.37 \mathrm{~Hz}$. From (10), this frequency is close to the undamped natural frequency of the interarea mode. 


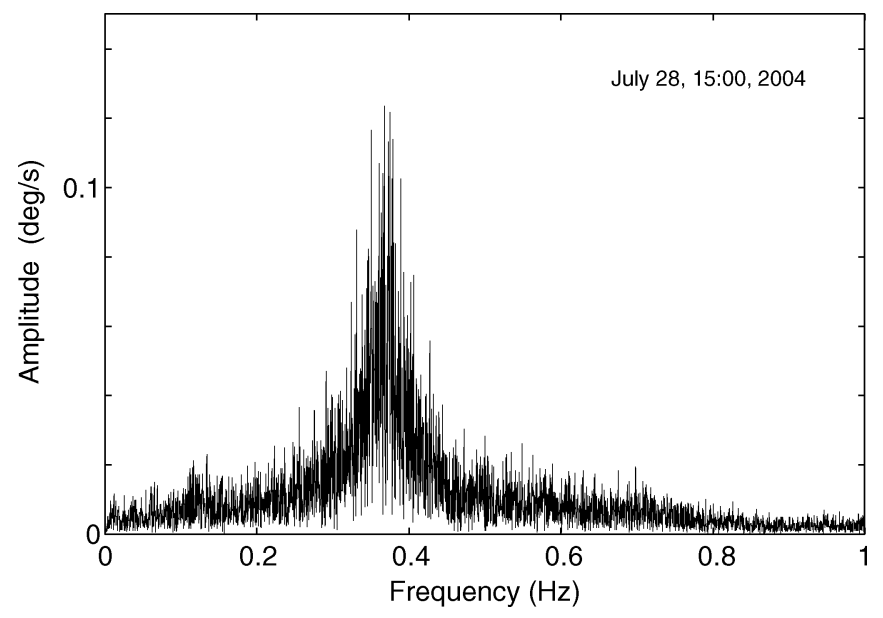

Fig. 5. Fourier spectrum for measurement $d \delta / d t$ in Fig. 2(b).

\section{ESTIMATION OF EIgENVALUE}

\section{A. Description of Method}

Comparison of Figs. 2 and 5 with Figs. 3 and 4 shows that the interarea mode is well described by (3). The Fourier spectra in Figs. 4 and 5 contain information on $\omega$ and $d$ of (3). If these parameters are known, the eigenvalue of the interarea mode is given by

$$
\lambda=-d \pm j \omega \sqrt{1-\left(\frac{d}{\omega}\right)^{2}} \simeq-d \pm j \omega .
$$

We estimate $\omega$ and $d$ by (8) from the Fourier spectra. The amplitude $c_{i}$ is obtained by FFT. However, $A_{i}$ is unknown; therefore, we use a curve-fitting method. We replace $A_{i}$ with a constant $A$, define a function

$$
g\left(\omega_{i}\right)=\frac{A}{\sqrt{\left(\omega_{i}^{2}-\omega^{2}\right)^{2}+4 d^{2} \omega_{i}^{2}}}
$$

and search for $\omega, d$, and $A$, which minimize

$$
I=\sum_{i=0}^{\frac{m}{2}}\left[c_{i}-g\left(\omega_{i}\right)\right]^{2} .
$$

A procedure of minimizing $I$ is described in Appendix B.

\section{B. Application of Method}

Fig. 6 shows an example of estimation. The Fourier spectrum in Fig. 4(b) was generated by simulation for $f=0.37 \mathrm{~Hz}$ and $d=0.1$, where $f=\omega / 2 \pi$. The estimated values from the spectrum are $f=0.36968, d=0.10722$, and $A=0.00062$. The estimated values agree well with the given values. Substituting them into (12) gives a smooth line in Fig. 6. For comparison, we consider an average amplitude defined by

$$
\bar{c}_{j}=\frac{1}{2 k+1} \sum_{j=i-k}^{i+k} c_{i}
$$

where $k$ is 15 in Fig. 6. The curve by (12) fits the averaged spectrum closely.

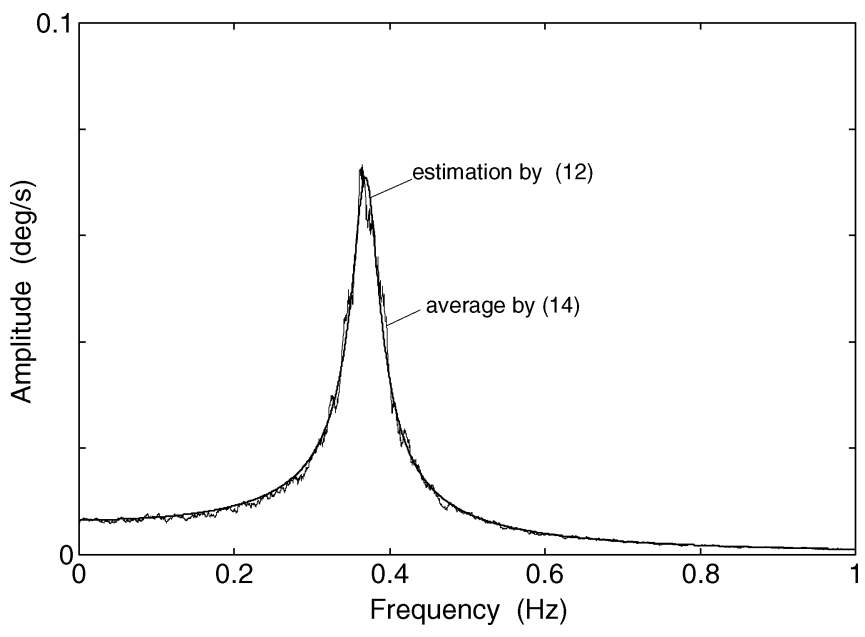

Fig. 6. Estimation of eigenvalue for simulation in Fig. 4(b).

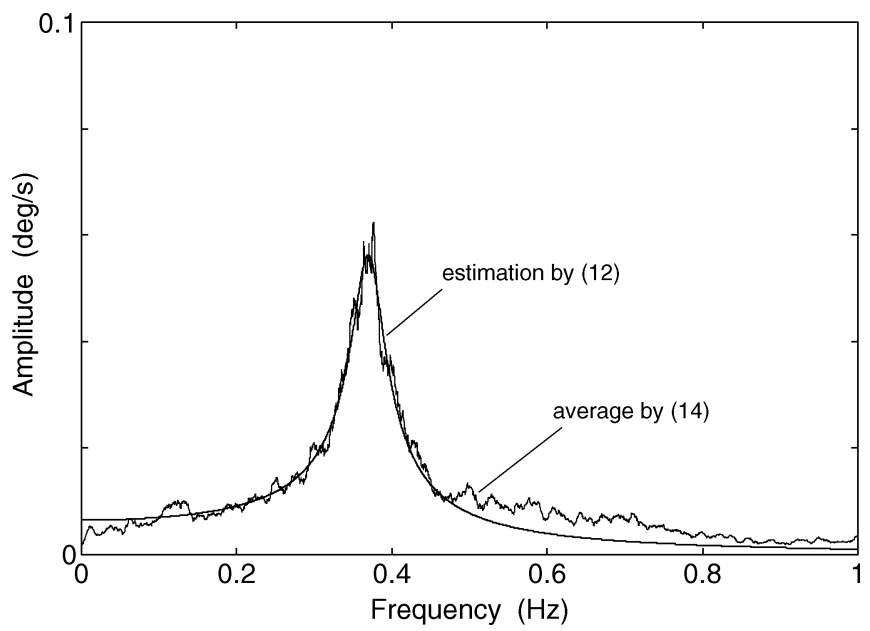

Fig. 7. Estimation of eigenvalue for measurement in Fig. 5.

Fig. 7 shows another example of estimation. The Fourier spectrum in Fig. 5 was obtained for the measurement in Fig. 2(b). The estimated values from the spectrum are $f=0.37057 \mathrm{~Hz}, d=0.13467$, and $A=0.00062$. The damped natural frequency by (11) shifts a little from 0.37057 to $0.36995 \mathrm{~Hz}$. The curve by (12) fits well the average amplitude $\bar{c}_{i}$, where $k$ is 15 . There is some discrepancy between them in the range over $0.5 \mathrm{~Hz}$, however. This discrepancy is brought by other oscillation modes. To avoid their influence on the estimation, we minimize (13) for $\omega_{i}$ in a limited range

$$
0 \leq \omega_{i}<2 \pi\left(f_{\max }+0.05\right)
$$

where $c_{i}$ takes its maximum at $f_{\max } \simeq f$.

\section{Stochastic Characteristics}

The proposed method is based on random variation of load, and it has a stochastic nature. To test its nature, we execute simulation of (3) and see variations of $f$ and $d$ in $24 \mathrm{~h}$. The number of samples and the sampling time are the same as in Fig. 3, i.e., $m=2^{15}$ and $\Delta t=0.1 \mathrm{~s}$. Since one time interval is 


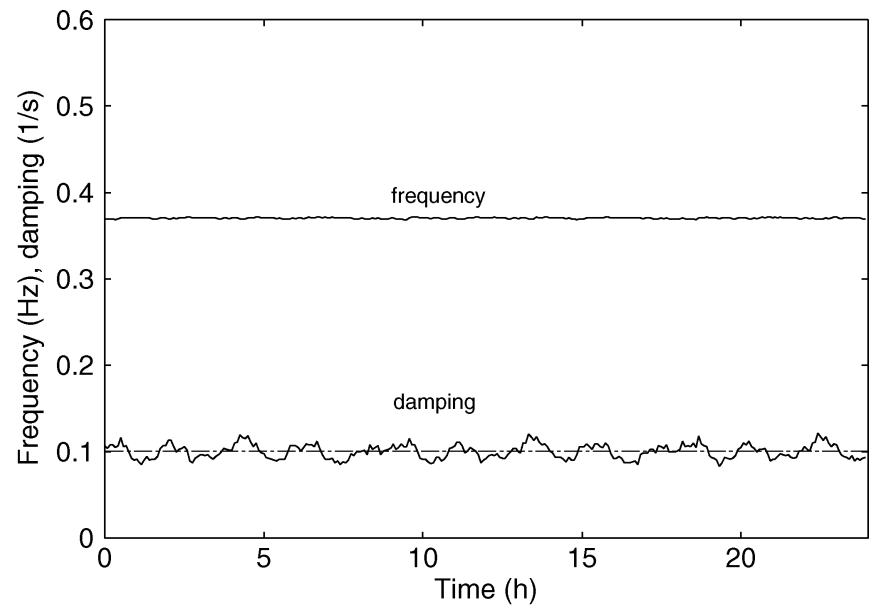

(a)

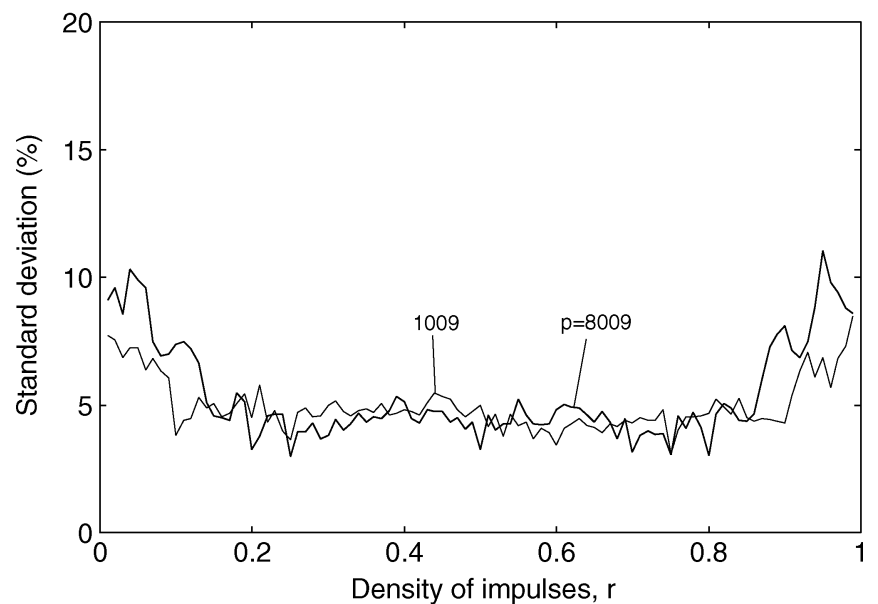

(b)

Fig. 8. Simulation. (a) Estimated values. (b) Standard deviation.

$2^{15} \times 0.1 \mathrm{~s}=54.6 \mathrm{~min}, 28$ intervals are necessary for the above simulation. The sequence of impulses $u$ are generated by (2). We use $j_{1}=1$ for the first interval but use $j_{n+1}$ as new $j_{1}$ for the second interval. This process is iterated for the succeeding intervals.

Fig. 8(a) shows estimated values of $f$ and $d$, where $f$ and $d$ of (3) are fixed at $0.37 \mathrm{~Hz}$ and 0.1 . The number of impulses are $n=0.9 \mathrm{~m}$. The estimation is made every $5 \mathrm{~min}$ with the next $2^{15}$ samples. Due to randomness of the impulses, the estimated values deviate from the true values. Their averages over $24 \mathrm{~h}$ are $0.370 \mathrm{~Hz}$ and 0.100 and are very close to the given values. The standard deviation $\sigma$ is $0.17 \%$ for $f$ and $8.11 \%$ for $d$. Thus, we can estimate $f$ very accurately, but we have some error in estimation of $d$. We can solve this problem by averaging estimated values over some time interval.

Fig. 8(b) shows the standard deviation for $d$ when the number of impulses changes as $n=r \times m$, where $r$ is the density of impulses, and $0 \leq r \leq 1$. Two sequences of impulses are generated by (2) with $p=8009$ and 1009 . There is no much difference between the results. The standard deviation is about $5 \%$ for $r=0.2 \sim 0.8$ and increases as $r \rightarrow 0$ or 1 . It remains less than $12 \%$, however. It is unknown which $\sigma$ applies to the real system. We examine this later.

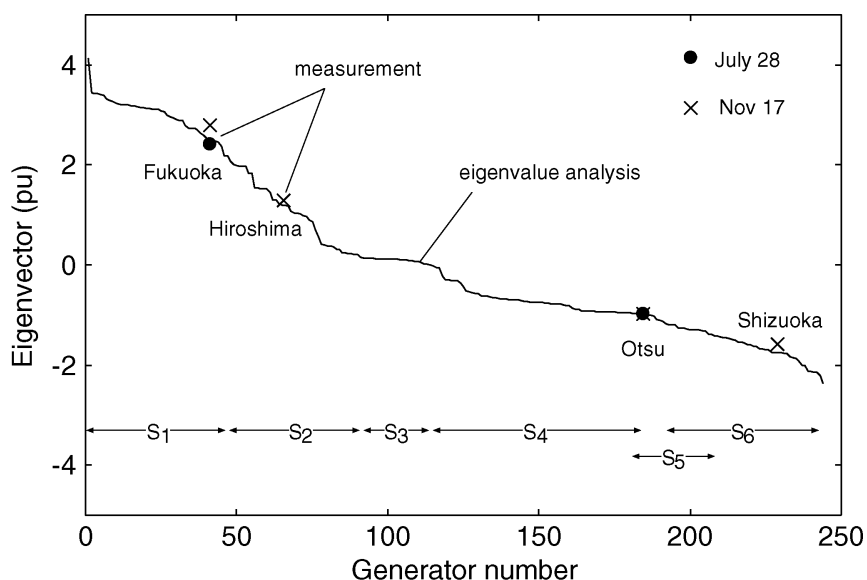

(a)

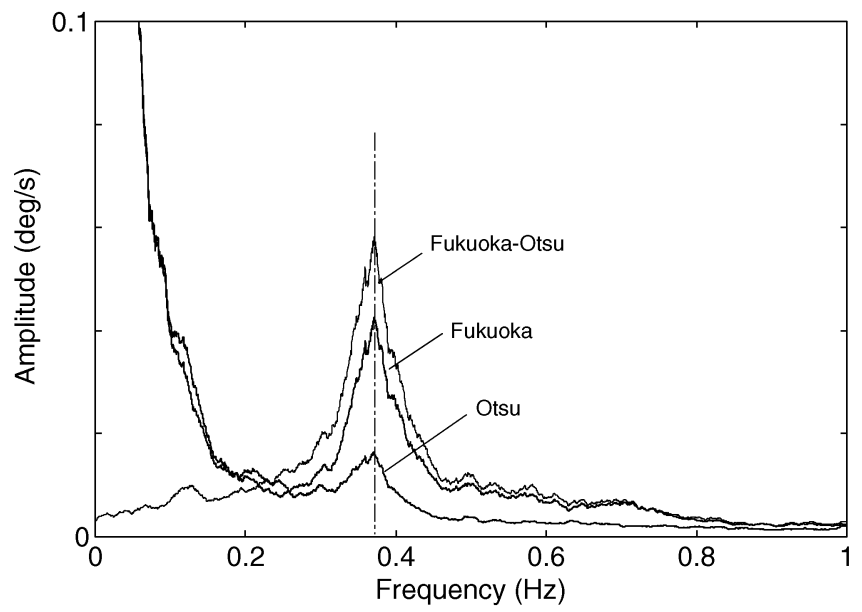

(b)

Fig. 9. Estimation of eigenvector. (a) Eigenvector. (b) Spectra.

\section{ESTIMATION OF EIGENVECTOR}

The eigenvalue of the interarea mode observed in Fig. 2 was estimated in Fig. 7. In this section, we consider to measure its eigenvector. Fig. 9(a) shows the eigenvector of the interarea mode obtained by eigenvalue analysis. The areas at 15:00 on July 28, 2004 are modeled with 244 generators. The generators are lined up from $S_{1}$ to $S_{6}$, while some overlap exists. The areas oscillate in two groups, i.e, $S_{1} \sim S_{3}$ and $S_{4} \sim S_{6}$. The eigenvector shows how each generator swings under the interarea mode. If we could measure this vector, it would be interesting. It is also useful for control of the mode [13].

Fig. 9(b) shows some Fourier spectra for the measurement in Fig. 2(b). The spectra are averaged by (14), where $k$ is 30 . In Fig. 7, we considered the difference between Fukuoka and Otsu. The result is shown again by a thin line. However, in this figure, we analyze them separately. The results are shown by two thick lines. We can see clear peaks at $0.37 \mathrm{~Hz}$, although some large components exist around $0 \mathrm{~Hz}$. The peak value is 0.0426 for Fukuoka and 0.0165 for Otsu. The sum is 0.0591 , which is very close to the peak value 0.0582 of Fukuoka-Otsu. This implies that Fukuoka and Otsu swing in the opposite direction. The ratio of 0.0426 to 0.0165 is 2.58 . By identifying generators near Fukuoka and Otsu, we place them on the eigenvector in Fig. 9(a). The measured results agree well with the eigenvalue 


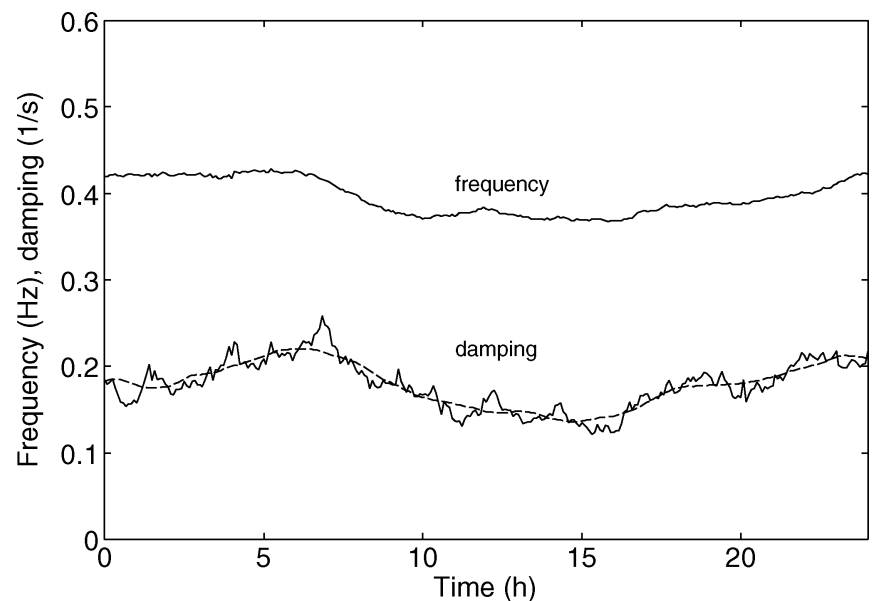

(a)

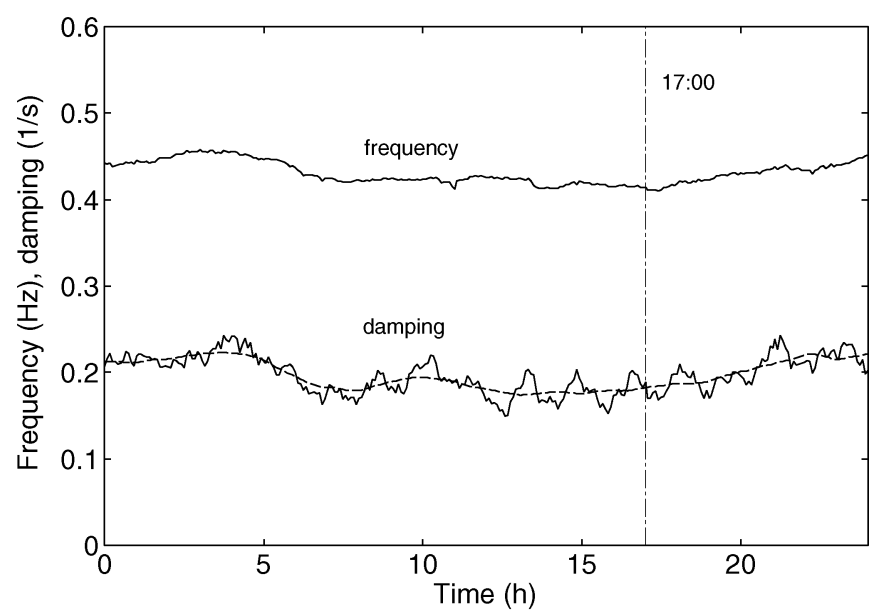

(b)

Fig. 10. Estimation. (a) July 28, 2004. (b) November 17, 2004.

analysis. In the figure, we also show the results for 15:00 of November 17, 2004, when we measured at four places. There is some change, but we can see good agreement between analysis and measurement. The eigenvector is stiff, but it varies with time and season.

\section{APPLICATION TO REAL SYSTEM}

Fig. 10 shows two estimation results. One is for July 28, 2004, when we have peak load of the year. The other is for November 17, 2004, when load is light. The estimation is made every 5 min. In both cases, the frequency varies slowly with time, but the damping fluctuates rapidly. This is similar to Fig. 8(a). The fluctuations are removed by taking the average over a time interval. The broken lines are for averages over $\pm 1.5 \mathrm{~h}$. It is not probable that the damping varies irrespective of the frequency. Therefore, this averaging may be necessary. The standard deviation $\sigma$ from the averaged value is $6.58 \%$ for July 28 and $6.04 \%$ for November 17. Judging from Figs. 2(c) and 8(b), the density of impulses is considered to be $0.9 \sim 1.0$.

Next, we check the accuracy of our method. As observed in Fig. 2, the interarea mode is constantly driven by small disturbances, and our method is based on this nature. Unlike Fig. 8, the true values of $f$ and $d$ are not given. However, due to a fault

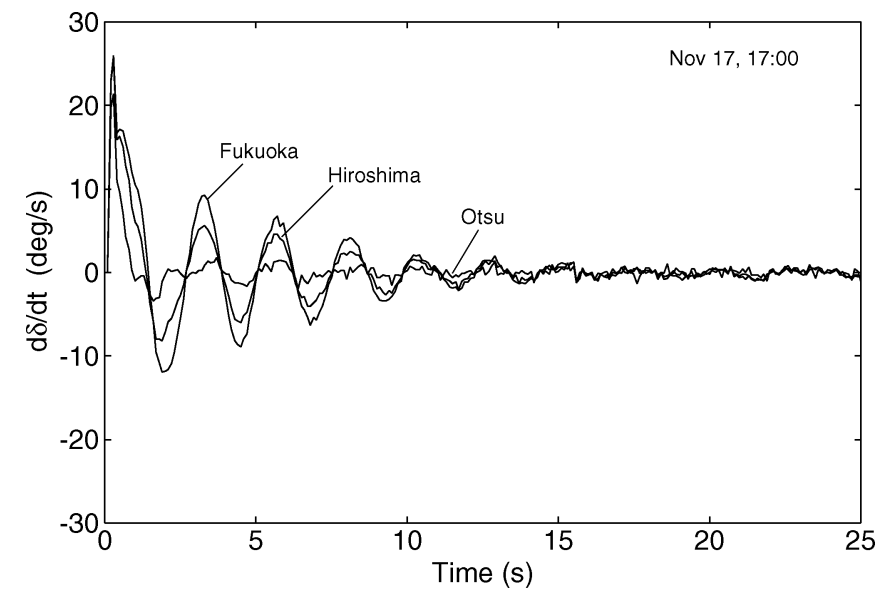

(a)

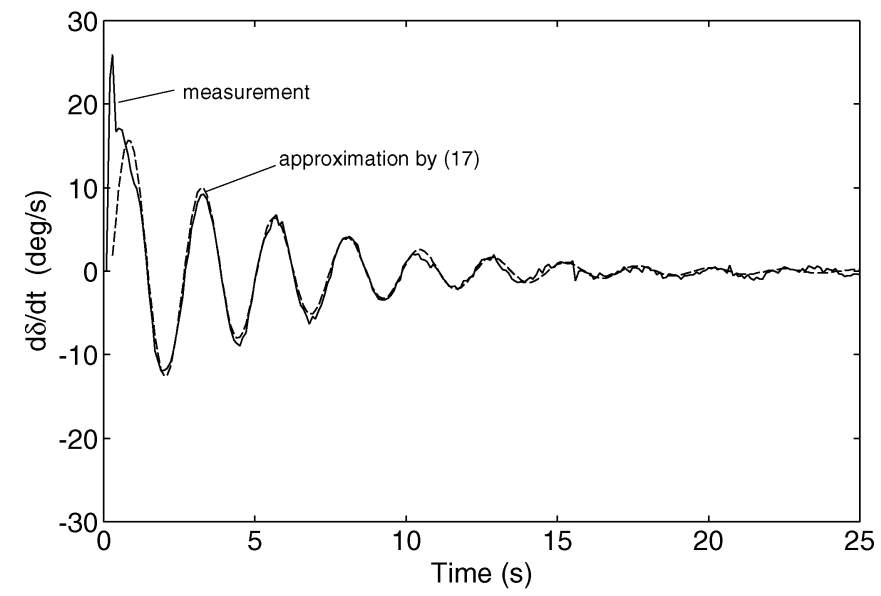

(b)

Fig. 11. Verification: November 17. (a) Measurement. (b) Curve-fitting.

or generator test, a relatively large disturbance is applied to the mode a few times per year. In such cases, small disturbances are negligible, and (3) reduces to

$$
\frac{d^{2} y}{d t^{2}}+2 d \frac{d y}{d t}+\omega^{2} y \simeq 0
$$

and $y$ varies according to

$$
y \simeq B e^{-d t} \sin (\omega t+\psi)
$$

until it reaches normal state, where $B$ and $\psi$ are constants. In these cases, we can determine $\omega$ and $d$ by curve-fitting of (17) on measured values of $y$. As a check, we compare these values with results estimated by our method.

Fig. 11 shows one example. A governor test was made near Shizuoka at 17:00 on November 17, 2004. Fig. 11(a) shows time variations of $y \equiv d \delta / d t$ at Fukuoka, Hiroshima, and Otsu, where Shizuoka is taken as reference. We can see familiar swing curves. Fig. 11(b) shows the swing curve between Fukuoka and Shizuoka. The values of $f$ and $d$ obtained by the curve-fitting in $t=1.5 \sim 15 \mathrm{~s}$ are $0.416 \mathrm{~Hz}$ and 0.187 . The approximation by (17) fits well the measured curve. The disturbance is not so large, and nonlinear effects are not seen. The estimated values in Fig. 10(b) are $0.414 \mathrm{~Hz}$ and 0.182 (averaged). They are close to the above values. 


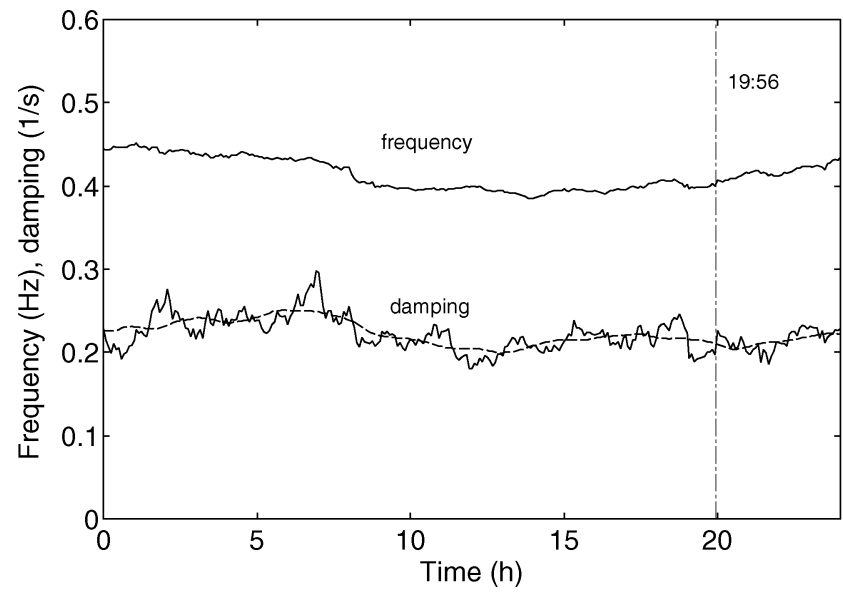

(a)

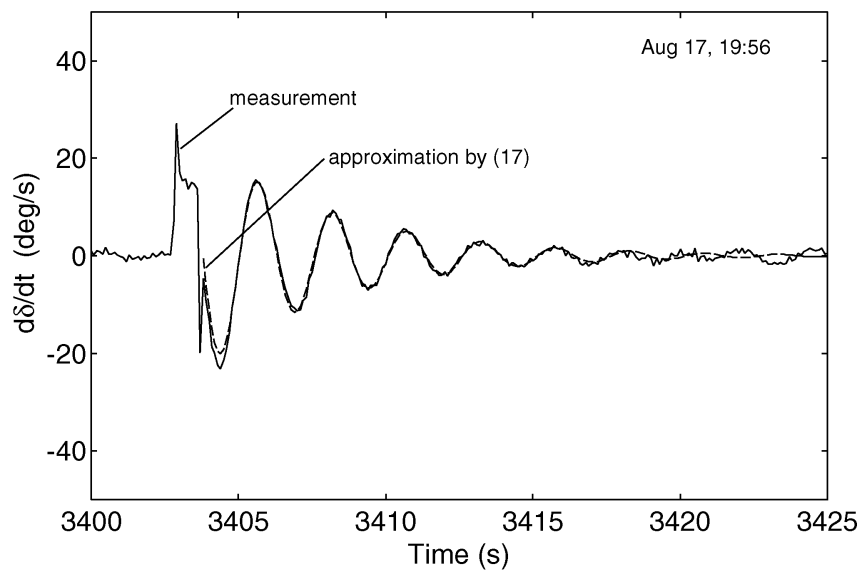

(b)

Fig. 12. Verification: August 17. (a) Estimation. (b) Curve-fitting.

Fig. 12 shows another example. There was a fault of a 500-kV system at 19:56 on August 17, 2004. Its location is the tie-line connecting $S_{1}$ and $S_{2}$. Fig. 12(a) shows estimated values of $f$ and $d$. We can see variations similar to Fig. 10. The values of $f$ and $d$ (averaged) at 19:56 are $0.400 \mathrm{~Hz}$ and 0.211. Fig. 12(b) shows the measured swing curve for the fault. The approximation by (17) fits well the measurement in $t=3405 \sim 3415 \mathrm{~s}$ and gives $f=0.396 \mathrm{~Hz}$ and $d=0.214$. Nonlinear effects are not seen, again. The values of $f$ and $d$ obtained by the two different methods agree well.

In the above examples, the interarea mode of interest was mainly excited, and we used them to check the validity of our method. However, other events may excite other modes and may influence our method. We can avert this problem by limiting the frequency range as (15) since the interarea mode is separated from the other modes, as shown in Fig. 7.

\section{MONITORING STABILITY}

Fig. 13 shows how the measured eigenvalue varies with the total generation in the areas. The eigenvalue by (11) is plotted every hour for May 9, July 28, and November 17 of 2004. The total generation was $37.6 \sim 51.7,44.6 \sim 64.6$, and $48.1 \sim$ $95.2 \mathrm{GW}$ for each day. With increase in the generation, the frequency lowers to $0.369 \mathrm{~Hz}$. The damping (averaged) also decreases with the total generation. The minimum damping is

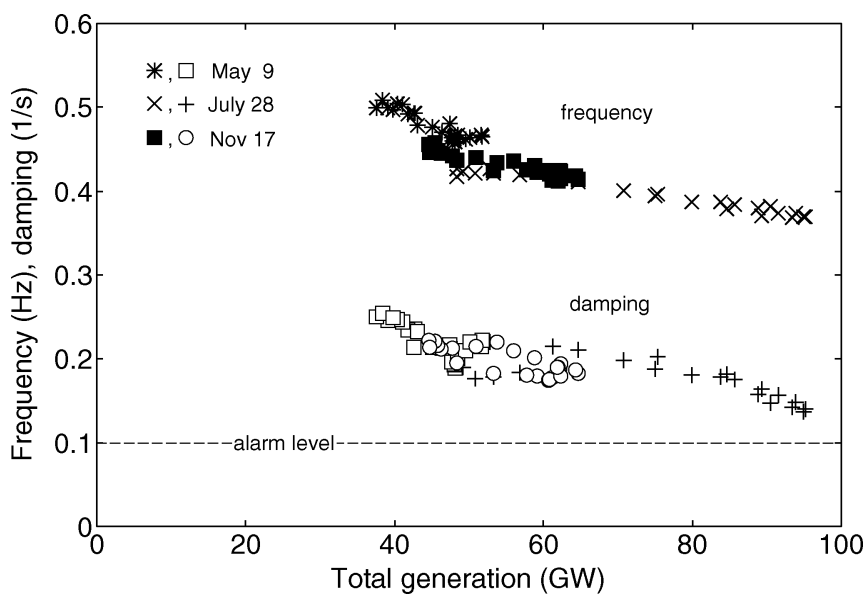

Fig. 13. Measured eigenvalue and total generation.

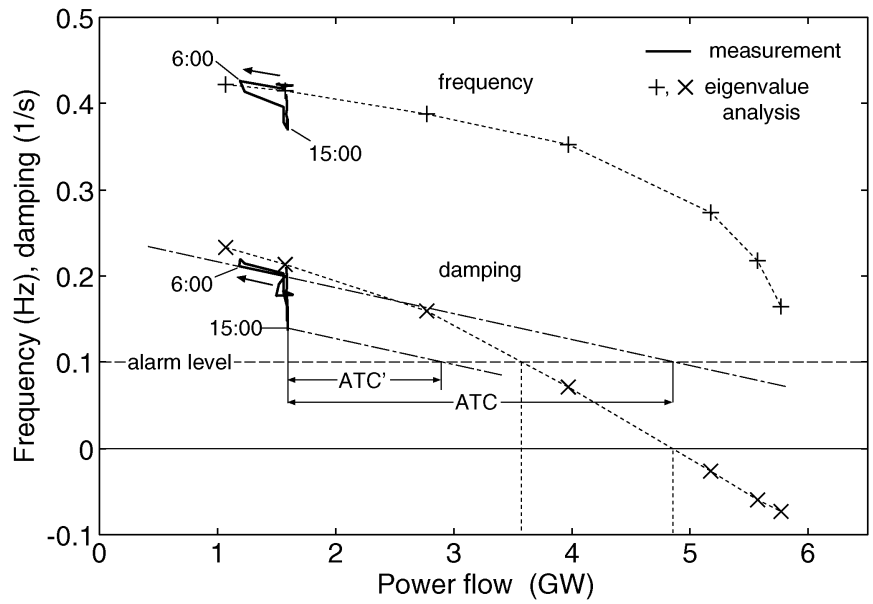

Fig. 14. Measured eigenvalue and power flow.

0.137 . This result implies that the stability of the interarea mode deteriorates with the total generation. It is desirable to maintain an appropriate level of stability, for example, $d=0.1$. We call this alarm level.

The eigenvalue also varies with power flow. Fig. 14 shows results of eigenvalue analysis for 15:00 of July 28, 2004. The power flow of the tie line connecting $S_{1}$ and $S_{2}$ in Fig. 1 was $1.57 \mathrm{GW}$. We change the flow, denoted by $P$, by adjusting the number of generators in $S_{1}$ and $S_{4}$. The frequency and damping lower with increase in the flow. The damping becomes zero at $4.84 \mathrm{GW}$. This is small signal stability limit. The damping reaches the alarm level, i.e., $d=0.1$ when the flow is $3.57 \mathrm{GW}$. The ATC based on this level is $2.00(=3.57-1.57) \mathrm{GW}$. If the flow varies freely as the eigenvalue analysis, it is easy to find the ATC. However, in the real system, the flow does not vary so much at present.

The power flow and the eigenvalue measured every hour on July 28 are shown in the figure. The power flow stayed around $1.57 \mathrm{GW}$ almost through the day. However, it reduced from $1.568 \mathrm{GW}(5: 00)$ to $1.184 \mathrm{GW}(6: 00)$. With this change, the damping increased from 0.20010 to 0.21193 . The total generation was 48.37 and $48.31 \mathrm{GW}$. The flow returned to $1.556 \mathrm{GW}$ at 9:00. The ratio of variations in $d$ and $P$ is

$$
\gamma=\frac{\Delta d}{\Delta P}=\frac{0.21193-0.20010}{1.568-1.184}=0.0308
$$


With this ratio, the ATC at 5:00 is estimated as

$$
\mathrm{ATC}=\frac{0.20010-0.1}{0.0308}=3.25 \mathrm{GW}
$$

This value is considerably greater than $2.00 \mathrm{GW}$. The damping decreases to 0.13995 at 15:00, and the ATC also reduces to

$$
\mathrm{ATC}^{\prime}=\frac{0.13995-0.1}{0.0308}=1.30 \mathrm{GW}
$$

Thus, ATC varies with time.

Two kinds of errors are considered to appear in the estimation of ATC. The first error comes from the fact that $d$ is a nonlinear function of $P$, as shown in Fig. 14. However, this error becomes small as $d$ approaches 0.1 and as $\Delta d$ becomes small. The second error comes from the accuracy of $\gamma$. One solution is to change $P$ by small amount $\Delta P$ and measure $\Delta d$ periodically. Further measurement and investigation will be required to obtain a more reliable value of ATC, however. This is left as a future work.

\section{CONCLUSION}

In this paper, we considered a method of monitoring an interarea oscillation mode in $60-\mathrm{Hz}$ areas of Japan. The results are summarized as follows.

1) Two PMUs set at Fukuoka and Otsu successfully measure deviation of phase angle due to the mode from $100-\mathrm{V}$ outlets on distribution systems during steady-state conditions.

2) The Fourier spectrum of the deviation shows a clear peak corresponding to the interarea mode. Its eigenvalue is accurately estimated by a curve-fitting method.

3) Its eigenvector is also estimated by separately calculating a Fourier spectrum for each measuring point. The estimation agrees well with the eigenvalue analysis.

4) The method is based on ambient noise caused by random load switching. It does not need an external signal to excite the mode and is applicable without disturbing the system.

5) The standard deviation of the estimation is about $6 \%$ for the real system. This deviation is removed by averaging estimates over $3 \mathrm{~h}$.

6) The method now uses 54.6 min of samples to obtain an estimate. It is desirable to reduce the number of samples.

7) The eigenvalue changes with the total generation and power flow in the areas. Monitoring it is useful to know stability limits and ATC.

\section{APPENDIX A}

\section{FOURIER TRANSFORM}

If $m(=2 N)$ samples of $x(t)$ are given at $t=j \Delta t$, where $j=0,1, \ldots, m-1$, they are represented by a finite Fourier series as follows:

$x(j \Delta t)=\frac{a_{0}}{2}+\sum_{i=1}^{N-1}\left(a_{i} \cos \frac{\pi j}{N} i+b_{i} \sin \frac{\pi j}{N} i\right)+\frac{a_{N}}{2} \cos \pi j$ where

$$
\begin{aligned}
& a_{i}=\frac{1}{N} \sum_{j=0}^{m-1} x(j \Delta t) \cos \frac{\pi i}{N} j \quad(i=0,1,2, \ldots, N) \\
& b_{i}=\frac{1}{N} \sum_{j=0}^{m-1} x(j \Delta t) \sin \frac{\pi i}{N} j \quad(i=1,2, \ldots, N-1) .
\end{aligned}
$$

The coefficients $a_{i}$ and $b_{i}$ are efficiently calculated by FFT. We rewrite (18) as

$$
x(j \Delta t)=\sum_{i=0}^{N} c_{i} \cos \left(\frac{\pi j}{N} i+\psi_{i}\right)
$$

where $c_{0}=a_{0} / 2, c_{N}=a_{N} / 2, \psi_{0}=\psi_{N}=0$, and

$$
c_{i}=\sqrt{a_{i}^{2}+b_{i}^{2}}, \quad \tan \psi_{i}=-\frac{b_{i}}{a_{i}} \quad(i=1,2, \ldots, N-1) .
$$

Further, (19) is transformed as

$$
\begin{aligned}
x(t) & =\sum_{i=0}^{N} c_{i} \cos \left(\frac{\pi i}{N \Delta t} j \Delta t+\psi_{i}\right) \\
& =\sum_{i=0}^{N} c_{i} \cos \left(\omega_{i} t+\psi_{i}\right)
\end{aligned}
$$

where

$$
\omega_{i}=\frac{\pi i}{N \Delta t}=\frac{2 \pi}{m \Delta t} i, \quad t=j \Delta t .
$$

Thus, (4) is derived.

\section{APPENDIX B MINIMIZATION}

The minimum point must satisfy

$$
\begin{aligned}
& \frac{\partial I}{\partial A}=-2 \sum_{i}\left(c_{i}-A h_{i}\right) h_{i}=0 \\
& \frac{\partial I}{\partial \omega}=-4 A \omega \sum_{i}\left(c_{i}-A h_{i}\right) h_{i}^{3}\left(\omega_{i}^{2}-\omega^{2}\right)=0 \\
& \frac{\partial I}{\partial d}=8 A d \sum_{i}\left(c_{i}-A h_{i}\right) h_{i}^{3} \omega_{i}^{2}=0
\end{aligned}
$$

where

$$
h_{i}=\left[\left(\omega_{i}^{2}-\omega^{2}\right)^{2}+4 d^{2} \omega_{i}^{2}\right]^{-\frac{1}{2}} .
$$

From (21), $A$ is given by

$$
A=\frac{\sum_{i} c_{i} h_{i}}{\sum_{i} h_{i}^{2}} .
$$

Equation (23) reduces to

$$
\sum_{i}\left(c_{i}-A h_{i}\right) h_{i}^{3} \omega_{i}^{2}=0
$$


From (22) and (25), we obtain

$$
\sum_{i}\left(c_{i}-A h_{i}\right) h_{i}^{3}=0 .
$$

We start from an initial approximation, for example, $f=$ $0.4 \mathrm{~Hz}$ and $d=0.2$, where $\omega=2 \pi f$. Since the value of $A$ is given by (24), $I$ is a function of $f$ and $d$. First, we minimize $I$ for $f$ and then for $d$ by changing them by 0.01 . We iterate this process until we reach the minimum point with the accuracy of 0.01 . Then, we apply the Newton-Raphson method to (25) and (26) to obtain a more accurate result.

\section{ACKNOWLEDGMENT}

The authors would like to thank Mr. T. Yanai for his help in eigenvalue analysis of the real $60-\mathrm{Hz}$ system of Japan.

\section{REFERENCES}

[1] N. Kakimoto, A. Nakanishi, and K. Tomiyama, "Instability of interarea oscillation mode by autoparametric resonance," IEEE Trans. Power Syst., vol. 19, no. 4, pp. 1961-1970, Nov. 2004.

[2] A. G. Phadke, "Synchronized phasor measurements in power systems," IEEE Comput. Appl. Power, vol. 6, no. 2, pp. 10-15, Apr. 1993.

[3] R. E. Wilson, "Methods and uses of precise time in power systems," IEEE Trans. Power Del., vol. 7, no. 1, pp. 126-132, Jan. 1992.

[4] R. O. Burnett, Jr., M. M. Butts, T. W. Cease, V. Centeno, G. Michel, R. J. Murphy, and A. G. Phadke, "Synchronized phasor measurements of a power system event," IEEE Trans. Power Syst., vol. 9, no. 3, pp. 1643-1650, Nov. 1994.

[5] K. E. Martin, G. Benmouyal, M. G. Adamiak, M. Begovic, R. O. Burnett Jr., K. R. Carr, A. Cobb, J. A. Kusters, S. H. Horowitz, G. R. Jensen, G. L. Michel, R. J. Murphy, A. G. Phadke, M. S. Sachdev, and J. S. Thorp, "IEEE standard for synchrophasors for power systems," IEEE Trans. Power Del., vol. 13, no. 1, pp. 73-77, Jan. 1998.

[6] R. Tsukui, P. Beaumont, T. Tanaka, and K. Sekiguchi, "Intranet-based protection and control," IEEE Comput. Appl. Power, vol. 14, no. 2, pp. 14-17, Apr. 2001.

[7] V. Centeno, J. De La Ree, A. G. Phadke, G. Michel, J. Murphy, and R. Burnett, "Adaptive out-of-step relaying using phasor measurement techniques," IEEE Comput. Appl. Power, vol. 6, no. 4, pp. 12-17, Oct. 1993.

[8] C. W. Taylor and D. C. Erickson, "Recording and analyzing the July 2 cascading outage," IEEE Comput. Appl. Power, vol. 10, no. 1, pp. 26-30, Jan. 1997.

[9] J. Hauer, D. Trudnowski, G. Rogers, B. Mittelstadt, W. Litzenberger, and J. Johnson, "Keeping an eye on power system dynamics," IEEE Comput. Appl. Power, vol. 10, no. 4, pp. 50-54, Oct. 1997.

[10] O. Faucon and L. Dousset, "Coordinated defense plan protects against transient instabilities," IEEE Comput. Appl. Power, vol. 10, no. 3, pp. 22-26, Jul. 1997.
[11] P. Denys, C. Counan, L. Hossenlopp, and C. Holweck, "Measurement of voltage phase for the French future defense plan against losses of synchronism," IEEE Trans. Power Del., vol. 7, no. 1, pp. 62-69, Jan. 1992.

[12] D. N. Kosterev, J. Esztergalyos, and C. A. Stigers, "Feasibility study of using synchronized phasor measurements for generator dropping controls in the Colstrip system," IEEE Trans. Power Syst., vol. 13, no. 3, pp. 755-761, Aug. 1998.

[13] I. Kamwa, R. Grondin, and Y. Hébert, "Wide-area measurement based stabilizing control of large power systems-a decentralized/hierarchical approach," IEEE Trans. Power Syst., vol. 16, no. 1, pp. 136-153, Feb. 2001.

[14] J. F. Hauer, C. J. Demeure, and L. L. Scharf, "Initial results in Prony analysis of power system response signals," IEEE Trans. Power Syst., vol. 5, no. 1, pp. 80-89, Feb. 1990.

[15] D. J. Trudnowski, M. K. Donnelly, and J. F. Hauer, "A procedure for oscillatory parameter identification," IEEE Trans. Power Syst., vol. 9, no. 4, pp. 2049-2055, Nov. 1994.

[16] T. Hashiguchi, M. Yoshimoto, Y. Mitani, O. Saeki, and K. Tsuji, "Analysis of generator oscillation characteristics based on multiple synchronized phasor measurements," IEEJ Trans. Power Energy, vol. 123, no. 12, pp. 1471-1477, Dec. 2003.

[17] T. E. Hull and A. R. Dobell, "Random number generators," SIAM Rev., vol. 4, no. 3, pp. 230-254, 1962.

[18] J. W. Cooley and J. W. Tukey, "An algorithm for the machine calculation of complex Fourier series," Math. Comput., vol. 19, pp. 297-301, 1965.

Naoto Kakimoto (M'78) was born in Japan in 1952. He received the Ph.D. degree in electrical engineering from Kyoto University, Kyoto, Japan, in 1982.

$\mathrm{He}$ is presently an Associate Professor at Kyoto University. His research interests are power system stabilities.

Masahiro Sugumi was born in Japan in 1982. He received the B.E. degree in electrical engineering from Kyoto University, Kyoto, Japan, in 2005. He is presently working toward the graduate degree at Kyoto University.

His research interests are power system stabilities.

Tohru Makino was born in Japan in 1982. He received the B.E. degree in electrical engineering from Kyoto University, Kyoto, Japan, in 2005. He is presently working toward the graduate degree at Kyoto University.

His research interests are power system stabilities.

Katsuyuki Tomiyama was born in Japan in 1945. He received the Ph.D. degree in electrical engineering from Kyoto University, Kyoto, Japan, in 2000.

$\mathrm{He}$ is a Chief Researcher at Kansai Electric Power Company, Osaka, Japan. Dr. Tomiyama is a member of the IEE of Japan. 